\title{
A REACTION INFILTRATION PROBLEM: CLASSICAL SOLUTIONS
}

\author{
by JOHN CHADAM, ${ }^{1}$ XINFU CHEN, ${ }^{2}$ ROBERTO GIANNI ${ }^{3}$ \\ and RICCARDO RICCI ${ }^{4}$
}

(Received 19th June 1995)

In this paper, we consider a reaction infiltration problem consisting of a parabolic equation for the concentration, an elliptic equation for the pressure, and an ordinary differential equation for the porosity. Existence and uniqueness of a global classical solution is proved for bounded domains $\Omega \subset \mathbf{R}^{N}$, under suitable boundary conditions.

AMS subject classifications (1991). 35M10, 35Q35.

\section{Introduction}

Here we consider the mathematical problem, introduced in [5] in order to study the infiltration of a fluid in a porous medium whose porosity changes due to the reaction of the porous matrix with the fluid. Part of the porous medium is then dissolved into the fluid and transported by the fluid flux. In this way the porosity and the concentration of the compound change with time and, in turn, influence the fluid motion.

Denote by $\varphi, u$, and $p$ the porosity of the medium, the concentration of the dissolved component, and the pressure in the fluid, respectively. Then the conservation law for the concentration, the conservation law for the mass of the fluid, and the dynamics of the dissolution of the porous matrix give rise to the following system of partial differential equations (see [5] for a detailed derivation)

$$
\begin{aligned}
\frac{\partial}{\partial t}(\varphi u) & =\nabla(D(\varphi) \nabla u+u K(\varphi) \nabla p)+\frac{\partial}{\partial t} \varphi, \\
\nabla(K(\varphi) \nabla p) & =\frac{\partial}{\partial t} \varphi \\
\frac{\partial}{\partial t} \varphi & =\left[\left(\varphi_{\mathrm{f}}-\varphi\right)_{+}\right]^{2 / 3}\left(u_{\mathrm{oq}}-u\right)
\end{aligned}
$$

'The author is partially supported by N.S.E.R.C. (Canada).

2 The author was partially supported by the National Science Foundation Grant DMS-9200459 (USA).

${ }^{3}$ The author is partially supported by M.U.R.S.T. (Italy).

${ }^{4}$ The author is partially supported by M.U.R.S.T. (Italy). 
where $t$ denotes the time, $\nabla$ is the spatial gradient, $D$ and $K$ are, respectively, the diffusivity and the permeability, $f_{+} \equiv \max \{f, 0\}$ is the positive part of $f, \varphi_{\mathrm{f}}$ and $u_{\mathrm{eq}}$ are positive constants representing the final porosity and the equilibrium concentration, respectively.

Here we consider the problem of solving (1.1)-(1.3) in $Q_{T}=\Omega \times(0, T)$ (where $\Omega \subset \mathbf{R}^{N}$ is a smooth bounded domain) with initial and boundary conditions

$$
\begin{gathered}
u(x, 0)=u_{0}(x), \quad x \in \Omega, \\
\varphi(x, 0)=\varphi_{0}(x), \quad x \in \Omega, \\
\frac{\partial u}{\partial n}(x, t)=0, \quad(x, t) \in \partial \Omega \times[0, T], \\
p(x, t)=p_{1}(x, t), \quad(x, t) \in \partial \Omega \times[0, T] .
\end{gathered}
$$

We are interested in global (in time) classical solutions of this problem. For $\Omega \subset \mathbf{R}^{1}$, the existence of a unique global classical solution was proved in [7], where the proof of the existence and uniqueness of a local (in time) classical solution was also established for any space dimension.

For $N=2$, global existence of a classical solution was proved in [4]. In this case the proof required the assumption of vanishing flux for the concentration, i.e. condition (1.6).

Here we extend the results obtained for dimension two to any spatial dimension.

Throughout this paper we use the notations of [10] for function spaces and their norms. In particular $H^{s}\left(Q_{T}\right), s \in \mathbf{R}$ is defined as in [10] (with "parabolic" anisotropic distance). We use also the space $C^{s}\left(Q_{T}\right)$ which is defined similarly but with the euclidean isotropic distance in $Q_{T}$.

\section{Hölder continuity}

In this section we prove the Hölder continuity of the concentration $u$. For this let us consider the equation

$$
\begin{gathered}
\varphi \frac{\partial}{\partial t} u=\nabla(D \nabla u)+K \nabla p \nabla u+f, \quad \text { in } Q_{T}=\Omega \times(0, T) \\
u(x, 0)=u_{0}(x, 0), \quad x \in \Omega \\
\frac{\partial u}{\partial n}(x, t)=0, \quad(x, t) \in \Gamma_{N}^{u} \times[0, T] \\
u(x, t)=u_{0}(x, t), \quad(x, t) \in \Gamma_{D}^{u} \times[0, T]
\end{gathered}
$$

where $\Gamma_{D}^{u}=\partial \Omega \backslash \Gamma_{N}^{u}$ and $u_{0}$ is the restriction of a $C^{1}\left(\bar{Q}_{T}\right)$ function. We also assume that 
the coefficients $\varphi, D, K$ and $f$ are measurable functions satisfying

$$
\begin{aligned}
& 0<\frac{1}{C_{0}} \leq \varphi, D, K \leq C_{0}, \\
&|f| \leq C_{0}, \\
&\left|\varphi_{t}\right| \leq C_{0},
\end{aligned}
$$

and the function $p \in L^{\infty}\left(0, T ; H^{1}(\Omega)\right)$ is a solution of

$$
\begin{gathered}
\nabla(K \nabla p)=g, \\
K \frac{\partial p}{\partial n}(x, t)=p_{N}(x, t), \quad(x, t) \in \Gamma_{N}^{p} \times[0, T], \\
p(x, t)=p_{D}(x, t), \quad(x, t) \in\left(\partial \Omega \backslash \Gamma_{N}^{p}\right) \times[0, T],
\end{gathered}
$$

where $g \in L^{\infty}\left(Q_{r}\right)$. We assume that $p_{N}$ is a measurable bounded function on $\partial \Omega \times[0, T]$ and $p_{D}$ is the restriction of a $C^{1}\left(\bar{Q}_{T}\right)$ function. If $\Gamma_{D}^{p}=\partial \Omega \backslash \Gamma_{N}^{p}=\emptyset$, we assume that $p_{N}$ contains a free term, depending only on time, in order to satisfy

$$
\int_{\Omega} p_{N}(\cdot, t)=\int_{\Omega} \varphi_{t}(\cdot, t)
$$

for every $t \in[0, T]$, and we choose $p$ such that

$$
\int_{\Omega} p(x, t)=0
$$

for every $t \in[0, T]$.

Theorem 2.1. Assume that $u \in L^{2}\left(0, T ; H^{1}(\Omega)\right) \cap L^{\infty}\left(Q_{T}\right)$ is a nonnegative solution of (2.1)-(2.4). Then there exist constants $\alpha \in(0,1)$ and $C>0$ such that

$$
|u|_{Q T}^{(x)} \leq C \text {. }
$$

The proof of the theorem follows the path of the proof in [10, Chapter II, §7]. However some modifications of the original proof are needed. For the reader's convenience we provide here most of the details. In the sequel $C$ will denote any constant depending on given data.

Lemma 2.1. Under the foregoing assumptions, we have

$$
\|p\|_{L^{\infty}\left(0, T ; C^{\beta}(\hat{\Omega})\right)} \leq C
$$

for some $\beta \in(0,1)$. 
Proof. It follows from the classical theory of elliptic equation in divergence form, see [9].

Lemma 2.2. For any given $\varepsilon>0$, there exists $\rho_{0}\left(C_{0}, \Omega, \Gamma_{D}^{p}\right)>0$ such that, if $\operatorname{spt}(\zeta) \subset B_{\rho_{0}}\left(x_{0}\right)$, for some $x_{0} \in \bar{\Omega}$, then

$$
\begin{aligned}
\int_{\Omega} K|\nabla p|^{2}(u-k)_{ \pm}^{2} \zeta^{2} & \leq \varepsilon \int_{\Omega}\left|\nabla(u-k)_{ \pm}\right|^{2} \zeta^{2} \\
& +C_{\varepsilon} \int_{\Omega}(u-k)_{ \pm}^{2}\left(\zeta^{2}+|\nabla \zeta|^{2}\right), \quad \text { for any } t \in[0, T]
\end{aligned}
$$

where $k \in \mathbf{R}$ is arbitrary, $(u-k)_{ \pm} \equiv \max \{ \pm(u-k), 0\}, \zeta$ is any cut-off function and $\operatorname{spt}(\zeta)$ is the support of $\zeta$.

Proof. Multiplying equation $(2.8)$ by $\left(p-p_{0}\right)(u-k)_{ \pm}^{2} \zeta^{2}$, where $p_{0}$ is a function to be determined in the following, we obtain

$$
\begin{aligned}
0 & =\int_{\Omega} K \nabla p \nabla\left(\left(p-p_{0}\right)(u-k)_{ \pm}^{2} \zeta^{2}\right)+\int_{\Omega} g\left(p-p_{0}\right)(u-k)_{ \pm}^{2} \zeta^{2} \\
& -\int_{\partial \Omega} K \frac{\partial p}{\partial n}\left(p-p_{0}\right)(u-k)_{ \pm}^{2} \zeta^{2} \\
& \equiv I+I I-I I I .
\end{aligned}
$$

We can estimate $I$ and $I I$ as follows:

$$
\begin{aligned}
I & =\int_{\Omega} K|\nabla p|^{2}(u-k)_{ \pm}^{2} \zeta^{2}-\int_{\Omega} K \nabla p \nabla p_{0}(u-k)_{ \pm}^{2} \zeta^{2} \\
& +\int_{\Omega} K\left(p-p_{0}\right) \nabla p\left[2(u-k)_{ \pm} \zeta^{2} \nabla(u-k)_{ \pm}+2(u-k)_{ \pm}^{2} \zeta \nabla \zeta\right] \\
& \geq \frac{1}{2} \int_{\Omega} K|\nabla p|^{2}(u-k)_{ \pm}^{2} \zeta^{2}-C\left\|\nabla p_{0}\right\|_{L^{\infty}(\Omega)} \int_{\Omega}(u-k)_{ \pm}^{2} \zeta^{2} \\
& -C\left\|p-p_{0}\right\|_{L^{\infty}\left(\beta_{p_{0}}\left(x_{0}\right) \cap \Omega\right)} \int_{\Omega}\left[\left|\nabla(u-k)_{ \pm}\right|^{2} \zeta^{2}+(u-k)_{ \pm}^{2}|\nabla \zeta|^{2}\right] \\
|I I| & \leq\|g\|_{L^{\infty}(\Omega)}\left\|p-p_{0}\right\|_{L^{\infty}\left(B_{\rho_{0}}\left(x_{0}\right) \cap \Omega\right)} \int_{\Omega}(u-k)_{ \pm}^{2} \zeta^{2} .
\end{aligned}
$$

To estimate $I I I$, we consider two cases:

(i) $\operatorname{dist}\left(x_{0}, \Gamma_{D}^{p}\right)>\rho_{0} ;$ (ii) $\operatorname{dist}\left(x_{0}, \Gamma_{D}^{p}\right) \leq \rho_{0}$.

In the first case, we take $p_{0}=p\left(x_{0}, t\right)$ which behaves like a constant in the above 
computation. Then it follows that

$$
\left|\nabla p_{0}\right|=0, \quad\left\|p-p_{0}\right\|_{L^{\infty}\left(B_{\rho_{0}}\left(x_{0}\right) \cap \Omega\right)} \leq \rho_{0}^{\beta}\|p\|_{L^{\infty}(0, T ; C \beta(\Omega))} \leq C \rho_{0}^{\beta}
$$

and then, using the imbedding $H^{1}(\Omega) \hookrightarrow L^{2}(\partial \Omega)$

$$
\begin{aligned}
|I I I| & =\left|\int_{\curvearrowright \Omega \mid \Gamma_{D}^{\rho}} p_{N}(x, t)\left(p-p_{0}\right)(u-k)_{ \pm}^{2} \zeta^{2}\right| \leq C \rho_{0}^{\beta}\left\|(u-k)_{ \pm} \zeta\right\|_{L^{2}(\Omega \Omega)}^{2} \\
& \leq C \rho_{0}^{\beta}\left\|(u-k)_{ \pm} \zeta\right\|_{H^{\prime}(\Omega)}^{2} \leq C \rho_{0}^{\beta} \int_{\Omega}\left[\left|\nabla(u-k)_{ \pm}\right|^{2} \zeta^{2}+(u-k)_{ \pm}^{2}\left(\zeta^{2}+|\nabla \zeta|^{2}\right)\right] .
\end{aligned}
$$

Combining this with the estimates for $I$ and $I I$, we finally obtain

$$
\begin{aligned}
\int_{\Omega} K|\nabla p|^{2}(u-k)_{ \pm}^{2} \zeta^{2} & \leq C \rho_{0}^{\beta} \int_{\Omega}\left|\nabla(u-k)_{ \pm}\right|^{2} \zeta^{2} \\
& +C \int_{\Omega}(u-k)_{ \pm}^{2}\left(\zeta^{2}+|\nabla \zeta|^{2}\right),
\end{aligned}
$$

which gives the assertion of the lemma for $\rho_{0}$ small enough.

In the second case we take $\rho_{0}=p_{D}$. Then we have $\left\|\nabla p_{0}\right\| \leq C_{0}$ and $\left\|p-p_{0}\right\|_{L^{\infty}\left(B_{0}\left(x_{0}\right) \cap \Omega\right)} \leq C \rho_{0}^{\beta}$ since $\operatorname{dist}\left(x_{0}, \Gamma_{D}^{\rho}\right)<\rho_{0}$. Consequently the above estimates remain true, and we obtain the assertion of the lemma again.

Remark 2.1. The result of the above lemma is the first fundamental estimate for the proof of Theorem 2.1. It allows to circumvent the problem of the lack of regularity of the coefficient $K \nabla p$ which forbids the direct use of the classical regularity results for linear parabolic equations. A similar approach was used in [1] for proving the continuity of the solution of a mixed parabolic-elliptic system. In [1] the parabolic equation was allowed to be degenerate. In the nondegenerate case the corresponding solution is Hölder continuous, [6].

In the sequel, $\rho_{0}$ will always indicate the radius determined in the above lemma.

Lemma 2.3. There exist constants $v, C>0$ such that, for every $\zeta \in C^{\infty}\left(B_{\rho_{0}}\left(x_{0}\right) \times[0, T]\right)$ vanishing on the lateral surface (for any $x_{0} \in \bar{\Omega}$ ) and any $0 \leq t_{1} \leq t_{2} \leq T$, we have

$$
\begin{array}{r}
\left.\int_{\Omega} \varphi(u-k)_{ \pm}^{2} \zeta^{2}\right|_{t=t_{1}} ^{t=t_{2}}+v \int_{t_{2}}^{t_{2}} \int_{\Omega}\left|\nabla(u-k)_{ \pm}\right|^{2} \zeta^{2} \\
\leq C \int_{t_{1}}^{t_{2}} \int_{\Omega}\left[(u-k)_{ \pm}^{2}\left(\left|\zeta \zeta_{t}\right|+|\nabla \zeta|^{2}\right)+\chi_{\left((u-k)_{ \pm}>0 !\right.} \zeta^{2}\right],
\end{array}
$$


where $k$ is any constant chosen according to the following

$$
\begin{cases}k \in[\min \{u\}, \max \{u\}], & \text { if } \operatorname{spt}(\zeta) \cap \Gamma_{D}^{u} \times\left[t_{1}, t_{2}\right]=\{\emptyset\} \\ k \geq \max _{\operatorname{spu}\left(\zeta \cap \Gamma_{D}^{u} \times\left\{t_{1}, t_{2}\right]\right.} u_{0} \text { in }(u-k)_{+} & \text {if } \operatorname{spt}(\zeta) \cap \Gamma_{D}^{u} \times\left[t_{1}, t_{2}\right] \neq\{\emptyset\} \\ k \leq \max _{\operatorname{sp}\left(\zeta \cap \Gamma_{D}^{u} \times\left[t_{1}, t_{2}\right]\right.} u_{0} \text { in }(u-k)_{-} & \end{cases}
$$

Proof. We deal only with the "+" case, the "-" being similar. Multiplying both sides of equation (2.1) by $(u-k)_{+} \zeta^{2}$ and integrating over $\Omega$ we get:

$$
\begin{aligned}
0 & =\int_{\Omega} \varphi u_{i}(u-k)_{+} \zeta^{2}-\int_{\partial \Omega} D \frac{\partial u}{\partial n}(u-k)_{+} \zeta^{2}+\int_{\Omega} D \nabla u \nabla\left((u-k)_{+} \zeta^{2}\right) \\
& -\int_{\Omega} K \nabla p \nabla u(u-k)_{+} \zeta^{2}-\int_{\Omega} f(u-k)_{+} \zeta^{2} \\
& \geq \frac{1}{2} \frac{d}{d t} \int_{\Omega} \varphi(u-k)_{+}^{2} \zeta^{2}-\frac{1}{2} \int_{\Omega}\left(\varphi_{t} \zeta^{2}+2 \varphi \zeta \zeta_{t}\right)(u-k)_{+}^{2}-\int_{\partial \Omega} D \frac{\partial u}{\partial n}(u-k)_{+} \zeta^{2} \\
& +\int_{\Omega} D\left|\nabla(u-k)_{+}\right|^{2} \zeta^{2}-\int_{\Omega} D\left(\frac{1}{8}\left|\nabla(u-k)_{+}\right|^{2} \zeta^{2}+8(u-k)_{+}^{2}|\nabla \zeta|^{2}\right) \\
& -\int_{\Omega}\left(\frac{1}{8} D\left|\nabla(u-k)_{+}\right|^{2} \zeta^{2}+8 \frac{K^{2}}{D}|\nabla p|^{2}(u-k)_{+}^{2} \zeta^{2}\right)-C \int_{\Omega} \chi_{\left\{(u-k)_{+}>0\right\}} \zeta^{2}
\end{aligned}
$$

Since $D(\partial u / \partial n)(u-k)_{+} \zeta^{2}=0$ on $\partial \Omega$, and $\varphi_{t} \in L^{\infty}$, the assertion the lemma follows from Lemma 2.2.

Remark 2.2. Inequality (2.13) is the analogous of inequality (7.5) of Chapt. II of [10], which implies the appartenence to a De Giorgi parabolic class of the solution. The main difference is the presence of the factor $\varphi$ in the first integral. If the ratio $\varphi_{M} / \varphi_{m}$ of the supremum to the infimum of $\varphi$ is bounded from above by $1 / 4$, then the proof of [10] can be carried over without changes. In fact this would allow to choose (in the notation of [10]) $p=2$ in Lemma 7.1, p. 112, which is the smallest possible value for which the proof of Lemma 7.3 works.

In the following $\left(x_{0}, t_{0}\right)$ will denote an arbitrary point of $Q_{T}$. We define

$$
\begin{aligned}
& Q_{\rho, 0}=\left(\Omega \cap B_{\rho}\left(x_{0}\right)\right) \times\left(t_{0}-\theta \rho^{2}, t_{0}\right), \\
& \mu_{ \pm}(k, \rho, t)=\text { measure }\left\{x \in \Omega \cap B_{\rho}\left(x_{0}\right) \mid(u(x, t)-k)_{ \pm}>0\right\}, \\
& A_{ \pm}(k, \rho, \theta)=\int_{t_{0}-0 \rho^{2}}^{t_{0}} \mu_{ \pm}(k, \rho, t) d t=\text { measure }\left\{(x, t) \in Q_{\rho, 0} \mid(u(x, t)-k)_{ \pm}>0\right\}, \\
& M_{\rho, 0}=\sup _{Q_{\rho, 0}} u, \quad m_{\rho, 0}=\inf _{Q_{\rho, 0}} u, \\
& \omega_{\rho, 0}=M_{\rho, 0}-m_{\rho, 0}=\operatorname{osc}_{Q_{\rho, 0}} u,
\end{aligned}
$$




$$
\begin{aligned}
& M_{\rho, 0}^{0}= \begin{cases}-\infty & \text { if } B_{\rho}\left(x_{0}\right) \cap \Gamma_{D}^{u}=\emptyset, \\
\max _{\left(B_{\rho}\left(x_{0}\right) \cap \Gamma_{D}^{u}\right) \times\left[t_{0}-0 \rho^{2}, t_{0}\right]} u_{0} & \text { if } B_{\rho}\left(x_{0}\right) \cap \Gamma_{D}^{u} \neq \emptyset,\end{cases} \\
& m_{\rho, 0}^{0}= \begin{cases}\infty & \text { if } B_{\rho}\left(x_{0}\right) \cap \Gamma_{D}^{u}=\emptyset, \\
\min _{\left(B_{\rho}\left(x_{0}\right) \cap \Gamma_{D}^{u}\right) \times\left[t_{0}-\theta \rho^{2}, t_{0}\right]} u_{0} & \text { if } B_{\rho}\left(x_{0}\right) \cap \Gamma_{D}^{u} \neq \emptyset .\end{cases}
\end{aligned}
$$

Lemma 2.4. There exist $\theta_{0} \in(0,1)$ and $b_{0}>0$ such that, for any $0<\rho<\rho_{0}<1$, one of the following cases holds:

(i) $\operatorname{dist}\left(x_{0}, \Gamma_{D}^{u}\right)<\rho$,

(ii) $\omega_{\rho, 0_{0}} \leq \rho$,

(iii) $\mu_{+}\left(m_{\rho, 0_{0}}+\frac{1}{8} \omega_{\rho, 0_{0}}, \rho, t\right)>b_{0} \rho^{N}$, for all $t \in\left[t_{0}-\theta_{0} \rho^{2}, t_{0}\right]$,

(iv) $\mu_{-}\left(M_{\rho, 0_{0}}-\frac{1}{8} \omega_{\rho, 0_{0}}, \rho, t\right)>b_{0} \rho^{N}$, for all $t \in\left[t_{0}-\theta_{0} \rho^{2}, t_{0}\right]$.

Proof. Assume that (i) and (ii) do not hold. Then dist $\left(x_{0}, \Gamma_{D}^{u}\right)>\rho$ and hence any $k \in[\min u, \max u]$ is admissible in Lemma 2.3 if $\operatorname{spt}(\zeta) \subset B_{p}\left(x_{0}\right)$. Moreover $\omega_{\rho, 1} \geq \omega_{\rho, 00} \geq \rho$.

Let $\theta_{0}$ be a small constant, whose value will be fixed in the sequel. One of the following cases must hold, for $t_{1}=t_{0}-\theta_{0} \rho^{2}$ :

(a) $\int_{B_{\rho} \cap \Omega} \varphi\left(\cdot, t_{1}\right) \chi\left\{u \geq \frac{m_{\rho, \theta_{0}}+M_{\rho, \theta_{0}}}{2}\right\} \geq \frac{1}{2} \int_{B_{\rho} \cap \Omega} \varphi\left(\cdot, t_{1}\right)$,

(b) $\int_{B_{\rho} \cap \Omega} \varphi\left(\cdot, t_{1}\right) \chi\left\{_{\left.L \leq \frac{m_{p, \theta_{0}}+M_{p, \theta_{0}}}{2}\right\}} \leq \frac{1}{2} \int_{B_{p} \cap \Omega} \varphi\left(\cdot, t_{1}\right)\right.$.

We now show that (a) implies (iii) and (b) implies (iv), hence completing the proof. Without loss of generality, we assume that (b) holds and prove that (b) implies (iv).

Let $\sigma \in(0,1)$ be a small constant to be determined and $\zeta=\zeta(x) \in C_{0}^{\infty}\left(B_{\rho}\left(x_{0}\right)\right)$ a cutoff function satisfying

$$
0 \leq \zeta \leq 1, \quad \zeta=1 \text { in } B_{(1-\sigma) \rho}\left(x_{0}\right), \quad|\nabla \zeta| \leq \frac{2}{\sigma \rho}
$$

Applying Lemma 2.3 with arbitrary $t_{2}=t \in\left[t_{1}, t_{0}\right]$ and $k=\left(m_{\rho, 0_{0}}+M_{\rho, 0_{0}}\right) / 2$, we have

$$
\begin{aligned}
\left.\int_{\Omega} \varphi(\cdot, t)(u-k)_{ \pm}^{2} \zeta^{2}\right|_{t} & \leq\left.\int_{\Omega} \varphi(\cdot, t)(u-k)_{ \pm}^{2} \zeta^{2}\right|_{t_{1}} \\
& +C \int_{t_{1}}^{t} \int_{\Omega}\left[(u-k)_{ \pm}^{2}\left(\zeta^{2}+|\nabla \zeta|^{2}\right)+\zeta^{2}\right] \\
& \leq\left(M_{\rho, 0_{0}}-k\right)^{2} \frac{1}{2} \int_{B_{\rho} \cap \Omega} \varphi\left(\cdot, t_{1}\right)
\end{aligned}
$$




$$
\begin{aligned}
& +C\left[\left(M_{\rho, 0_{0}}-k\right)^{2} \frac{\rho^{N-2}}{\sigma^{2}}+\rho^{N}\right]\left(t-t_{1}\right) \\
& \leq \frac{\omega_{\rho, 0_{0}}^{2}}{8} \int_{B_{\rho} \cap \Omega} \varphi\left(\cdot, t_{1}\right)+C \theta_{0} \omega_{\rho, 0_{0}}^{2} \frac{\rho^{N}}{\sigma^{2}}
\end{aligned}
$$

since $M_{\rho, 0_{0}}-k=\frac{1}{2} \omega_{\rho, 0_{0}}>\frac{1}{2} \rho, t-t_{1} \leq \theta \rho^{2},(\rho<1)$. From the previous estimates (and the fact that $\left.M_{\rho, 0_{0}}-\frac{1}{8} \omega_{\rho, 0_{0}}-k=\frac{3}{8} \omega_{\rho, 0_{0}}\right)$ it follows that

$$
\begin{aligned}
& \int_{B_{(1-\sigma) \rho} \cap \Omega} \varphi(\cdot, t) \chi_{\left(u \leq M_{\rho, 0_{0}}-\frac{1}{8} \omega_{\rho, 0_{0}}\right)}=\int_{B_{(1-\sigma) \rho} \cap \Omega} \varphi(\cdot, t) \\
& -\int_{B_{(1-\sigma) \rho} \cap \Omega} \varphi(\cdot, t) \chi_{\left(u>M_{\rho, 0_{0}}-\frac{1}{8} \omega_{\rho, O_{0}}\right)} \\
& \geq \int_{B_{(1-\sigma) \rho} \cap \Omega} \varphi(\cdot, t)-\frac{1}{\left(M_{\rho, 0_{0}}-\frac{1}{8} \omega_{\rho, 0_{0}}-k\right)^{2}} \int_{B_{(1-\sigma) \rho} \cap \Omega} \varphi(\cdot, t)(u-k)_{+}^{2} \zeta^{2} \\
& \geq \int_{B_{(1-\sigma) \rho} \cap \Omega} \varphi(\cdot, t)-\frac{8}{9} \int_{B_{\rho} \cap \Omega} \varphi\left(\cdot, t_{1}\right)-C \theta_{0} \frac{\rho^{N}}{\sigma^{2}} .
\end{aligned}
$$

Since $\left|\varphi_{t}\right|<C_{0}$, we have

$$
\begin{aligned}
\int_{B_{(1-\sigma) \rho} \cap \Omega} \varphi(\cdot, t) & -\frac{8}{9} \int_{B_{\rho} \cap \Omega} \varphi\left(\cdot, t_{1}\right) \geq \frac{1}{9} \int_{B_{(1-\sigma) \rho} \cap \Omega} \varphi(\cdot, t) \\
& -\frac{8}{9} \int_{B_{(1-\sigma) \rho} \cap \Omega}\left|\varphi(\cdot, t)-\varphi\left(\cdot, t_{1}\right)\right|-\frac{8}{9} \int_{\left(B_{\rho} \backslash B_{(1-\sigma) \rho) \cap \Omega}\right.} \varphi(\cdot, t) \\
& \geq \frac{1}{C^{*}} \rho^{N}-C \rho^{N+2} \theta_{0}-C \sigma \rho^{N}
\end{aligned}
$$

Hence we have

$$
\int_{B_{(1-\sigma) \rho} \cap \Omega} \varphi(\cdot, t) \chi_{\left\{u \leq M_{\rho, 0_{0}}-\frac{1}{8} \omega_{\left.\rho, \theta_{0}\right)}\right.} \geq \frac{1}{C^{*}} \rho^{N}-C \rho^{N+2} \theta_{0}-C \sigma \rho^{N}-C \frac{\theta_{0} \rho^{N}}{\sigma^{2}} \geq \frac{\rho^{N}}{2 C^{*}}
$$

by taking $\sigma=1 / 4 C C^{*}$ and $\theta_{0}$ small enough. Since $\varphi \leq C_{0}$, the assertion of the lemma follows.

In the sequel we fix $\theta=\theta_{0}$ and we omit the subscript $\theta$ in all related quantities, e.g. $Q_{\rho, 0}=Q_{\rho}$, etc.

Lemma 2.5. There exists a constant $C>0$ such that for all $\rho \in\left(0, \rho_{0}\right), \sigma \in\left(0, \frac{1}{2}\right)$, and $k$ satisfying (2.14) 


$$
\begin{aligned}
&\|\|(u-k)_{ \pm}\left\|\left.\right|_{Q_{(1-\sigma) \rho}} ^{2} \equiv \sup _{t_{0}-\sigma(1-\sigma)^{2} \rho^{2}<i<t_{0}}\right\|(u-k)_{ \pm} \|_{L^{2}\left(B_{(1-\sigma) \rho} \cap \Omega\right)}^{2} \\
&+\left\|\nabla(u-k)_{ \pm}\right\|_{L^{2}\left(Q_{(1-\sigma) \rho} \cap \Omega\right)}^{2} \\
& \leq C\left[\frac{1}{\sigma^{2} \rho^{2}}\left\|(u-k)_{ \pm}\right\|_{Q_{\rho}}^{2}+A_{ \pm}(k, \rho)\right]
\end{aligned}
$$

Proof. It is enough to choose the cut-off function $\zeta(x, t) \in C^{\infty}\left(\mathbf{R}^{N+1}\right)$ in Lemma 2.3 such that $\zeta=0$ if $\left|x-x_{0}\right| \geq \rho$ or $t<t_{0}-\theta_{0} \rho^{2}, \zeta=1$ in $Q_{(1-\sigma) \rho}, 0 \leq \zeta \leq 1$ and $\left|\zeta_{t}\right|+|\nabla \zeta|^{2} \leq C / \sigma \rho^{2}$.

Lemma 2.6. Assume that $H_{0} \in\left(M_{\rho}-\frac{1}{4} \omega_{\rho}, M_{\rho}\right)$ (or $\left.H_{0} \in\left(m_{\rho}, m_{\rho}+\frac{1}{4} \omega_{\rho}\right)\right)$ and that $H_{0} \geq M_{\rho}^{0}\left(\right.$ or $\left.H_{0} \leq m_{\rho}^{0}\right)$. Define, for $n=1,2, \ldots$,

$$
\begin{aligned}
H_{n} & =M_{\rho}-\left(\frac{1}{2}+\frac{1}{2^{n}}\right)\left(M_{\rho}-H_{0}\right) \quad\left(\text { or } H_{n}=m_{\rho}+\left(\frac{1}{2}+\frac{1}{2^{n}}\right)\left(H_{0}-m_{\rho}\right)\right) \\
\rho_{n} & =\frac{1}{2}\left(\frac{1}{2}+\frac{1}{2^{n}}\right) \rho
\end{aligned}
$$

Then

$$
\frac{A_{+}\left(H_{n+1}, \rho_{n+1}\right)}{\rho^{N}} \leq C^{n}\left(\frac{A_{+}\left(H_{n}, \rho_{n}\right)}{\rho^{N}}\right)^{1+\frac{2}{N}}
$$

Or

$$
\frac{A_{-}\left(H_{n+1}, \rho_{n+1}\right)}{\rho^{N}} \leq C^{n}\left(\frac{A_{+}\left(H_{n}, \rho_{n}\right)}{\rho^{N}}\right)^{1+3}
$$

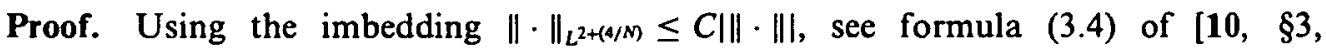
Chapt. 2, p. 75], and Lemma 2.5, we have

$$
\begin{aligned}
A_{+}\left(H_{n+1}, \rho_{n+1}\right) & \leq \frac{1}{\left(H_{n+1}-H_{n}\right)^{2+(4 / M)}} \int_{Q_{\rho_{n+1}}}\left(u-H_{n}\right)_{+}^{2+(4 / M)} \\
& \leq\left. C \frac{1}{\left(H_{n+1}-H_{n}\right)^{2+(4 / N)}}\left\|\left(u-H_{n}\right)_{+}\right\|\right|_{Q_{\rho_{n+1}}^{2+(4 / N)}} \\
& \leq C \frac{1}{\left(H_{n+1}-H_{n}\right)^{2+(4 / M)}}\left[\frac{1}{\left(\rho_{n}-\rho_{n+1}\right)^{2}}\left\|\left(u-H_{n}\right)_{+}\right\|_{Q_{\rho_{n}}}^{2}+A_{+}\left(H_{n}, \rho_{n}\right)\right]^{1+\frac{2}{n}} \\
& \leq C^{n} A_{+}\left(H_{n}, \rho_{n}\right)^{1+(2 / N)}
\end{aligned}
$$


where we made use of $\left\|\left(u-H_{n}\right)_{+}\right\|_{Q_{\rho_{n}}}^{2} \leq 4^{n+1}\left(H_{n+1}-H_{n}\right)^{2} A_{+}\left(H_{n}, \rho_{n}\right)$.

Lemma 2.7. We have

(i) If mes $\left(\{u(\cdot, t)<k\} \cap B_{\rho} \cap \Omega\right) \geq b_{0} \rho^{N}$ then, for all $l \geq k$

$$
(l-k) \mu_{+}(l, \rho, t) \leq C \rho\left\|\nabla(u(\cdot, t)-k)_{+}\right\|_{L^{2}\left(B_{\theta} \cap \Omega\right)}\left(\mu_{+}(k, \rho, t)-\mu_{+}(l, \rho, t)\right)^{1 / 2},
$$

(ii) If $H^{N-1}\left(B_{\rho} \cap \Gamma_{D}^{u}\right) \geq b_{0} \rho^{N-1}$ and $\left.u\right|_{B_{\rho} \cap \Gamma_{D}^{u}} \leq k$, then (2.15) holds. Here $H^{N-1}$ denotes the Hausdorff measure.

Proof. Part (i) of the lemma follows immediately from inequality (5.5) of [10, Chapt. II, p. 91], by means of the Hölder inequality. To prove (ii), one has to prove first an equivalent of the above mentioned inequality (5.5) when the set $\{u \leq k\}$ is localized on the boundary of $B_{\rho} \cap \Omega$. This is almost immediate if $B_{\rho} \cap \Omega=B_{\rho}$ since, in this case, the same proof as in [10] can be carried out simply integrating over the boundary of $B_{\rho}$. The general case can be reduced to this one if $\partial \Omega$ is regular enough and $\rho$ is sufficiently small.

Lemma 2.8. For every $\varepsilon>0$, there exists $S(\varepsilon)>0$ such that one of the following holds:

(1) $\omega_{\rho / 2} \leq 2^{s} \rho$

(2) $M_{\rho} \geq M_{2 \rho}^{0}+\frac{1}{4} \omega_{\rho}$

and $A_{+}\left(M_{\rho}-\frac{1}{2^{s}} \omega_{\rho / 2}, \frac{\rho}{2}\right) \leq \varepsilon\left(\frac{\rho}{2}\right)^{N+2}$

(3) $m_{\rho} \leq m_{2 \rho}^{0}-\frac{1}{4} \omega_{\rho}$

and $A_{-}\left(m_{\rho}+\frac{1}{2^{s}} \omega_{\rho / 2}, \frac{\rho}{2}\right) \leq \varepsilon\left(\frac{\rho}{2}\right)^{N+2}$

(if $\operatorname{dist}\left(x_{0}, \Gamma_{D}^{u}\right) \leq \rho$ then $2 \rho$ replaces $\rho$ in the inequalities involving $A_{+}$and $A_{-}$).

Proof. We are of course interested in the case in which (1) fails. First we consider the case dist $\left(x_{0}, \Gamma_{D}^{u}\right)>\rho$. By Lemma 2.4 we can assume, without loss of generality, that

$$
\mu_{-}\left(M_{\rho / 2}-\frac{1}{8} \omega_{\rho / 2}, \frac{\rho}{2}, t\right) \geq b_{0} \rho^{N} \quad \text { for every } t \in\left[t_{0}-\frac{\theta_{0}}{4} \rho^{2}, t_{0}\right]
$$

Hence, taking $l>k>M_{\rho / 2}-\frac{1}{8} \omega_{\rho / 2}$ in Lemma 2.7 (i), and from the estimate of Lemma 2.5 , we have 


$$
\begin{aligned}
A_{+}^{2}\left(l, \frac{\rho}{2}\right) & \leq \frac{C \rho^{2}}{(l-k)^{2}} \int_{t_{0}-\left(0_{0} / 4\right) \rho^{2}}^{t_{0}}\left\|\nabla(u-k)_{+}\right\|_{L^{2}\left(B_{\rho / 2} \cap \Omega\right)}^{2}\left(A_{+}\left(k, \frac{\rho}{2}\right)-A_{+}\left(l, \frac{\rho}{2}\right)\right) \\
& \leq \frac{C}{(l-k)^{2}}\left[\left\|(u-k)_{+}\right\|_{Q_{\rho}}^{2}+\rho^{N+4}\right]\left(A_{+}\left(k, \frac{\rho}{2}\right)-A_{+}\left(l, \frac{\rho}{2}\right)\right) \\
& \leq C\left(\frac{M_{\rho}-k}{l-k}\right)^{2} \rho^{N+2}\left(A_{+}\left(k, \frac{\rho}{2}\right)-A_{+}\left(l, \frac{\rho}{2}\right)\right)\left[1+\frac{\rho^{2}}{\left(M_{\rho}-k\right)^{2}}\right]
\end{aligned}
$$

Let us take $k_{n}=M_{\rho}-\frac{1}{8} \frac{1}{2^{n}} \omega_{\rho / 2}, n=0,1,2,3, \ldots, S-3$. Then, taking $k=k_{n}$, and $l=k_{n+1}$, we have that

$$
A_{+}^{2}\left(k_{n+1}, \frac{\rho}{2}\right) \leq 4 C \rho^{N+2}\left(A_{+}\left(k_{n}, \frac{\rho}{2}\right)-A_{+}\left(k_{n+1}, \frac{\rho}{2}\right)\right)\left[1+\left(8 \frac{2^{n} \rho}{\omega_{\rho / 2}}\right)^{2}\right]
$$

Since $\omega_{\rho / 2} \geq 2^{s} \rho$, adding up the above inequalities for $n=0,1,2, \ldots, S-3$, we obtain

$$
A_{+}^{2}\left(k_{s}, \frac{\rho}{2}\right) \leq \frac{C}{S-2} \rho^{N+2} A_{+}\left(k_{0}, \frac{\rho}{2}\right) \leq \frac{C}{S-2} \rho^{N+2} \rho^{N+2} .
$$

Taking $S$ large enough, we obtain

$$
A_{+}\left(k_{s}, \frac{\rho}{2}\right) \leq \varepsilon\left(\frac{\rho}{2}\right)^{N+2}
$$

which is the claim of the lemma.

We now consider the case dist $\left(x_{0}, \Gamma_{D}^{u}\right) \leq \rho$. Since

$$
\omega_{2 \rho}^{0} \equiv \operatorname{osc}_{\Gamma_{D}^{u} \cap \bar{Q}_{2 \rho}\left(x_{0}\right)} u_{0} \leq C \rho,
$$

we can assume, without loss of generality, $\omega_{\rho} \geq 2 \omega_{2 \rho}^{0}$, and therefore, either

or

$$
M_{\rho} \geq \max _{\Gamma_{D}^{n} \cap B_{2 \rho}\left(x_{0}\right)} u_{0}+\frac{1}{4} \omega_{\rho}
$$

$$
m_{\rho} \leq \min _{\Gamma_{D}^{n} \cap B_{2 \rho}\left(x_{0}\right)} u_{0}-\frac{1}{4} \omega_{\rho}
$$

Assume that the first case holds (the second one is analogous). We can now use the results of Lemma 2.7 (ii) and of Lemma 2.5. Then, proceeding as above, we show that there exists $S$ such that

$$
A_{+}\left(k_{s}, \rho\right) \leq \varepsilon(\rho)^{N+2}, \quad k_{s}=M_{2 \rho}-\frac{1}{2^{s}} \omega_{\rho}
$$


This completes the proof of lemma.

We now state an algebraic lemma whose proof can be found, for instance, in [10].

Lemma 2.9. If the sequence $\left\{y_{n}\right\}_{n=0}^{\infty}$ satisfies

$$
0 \leq y_{n+1} \leq C^{n} y_{n}^{1+(2 / N)}
$$

for some positive $C>1$, then there exists $\varepsilon>0$ depending on $C$, such that, if $y_{0}<\varepsilon$, then

$$
\lim _{n \rightarrow \infty} y_{n}=0 \text {. }
$$

In the sequel we fix the value of $\varepsilon$ in Lemma 2.8 in such a way that Lemma 2.9 can be applied.

Lemma 2.10. There exists $v \in(0,1)$ such that, for all $0<\rho<\rho_{0}$, we have

$$
\omega_{\rho / 4} \leq v \omega_{2 \rho}+C \rho
$$

Proof. Without loss of generality we can assume $\omega_{\rho / 4} \geq C \rho$. Let us first consider the case dist $\left(x_{0}, \Gamma_{D}^{u}\right)>\rho$. In this case, using Lemma 2.6, Lemma 2.8 and Lemma 2.9, we have that either

or

$$
M_{\rho / 4} \leq M_{\rho}-\frac{1}{2^{S+1}} \omega_{\rho / 2}
$$

$$
m_{\rho / 4} \geq m_{\rho}+\frac{1}{2^{S+1}} \omega_{\rho / 2}
$$

In both cases we have that

$$
\omega_{\rho / 4} \leq \omega_{\rho}-\frac{1}{2^{s+1}} \omega_{\rho / 2}
$$

from which we get

$$
\omega_{\rho / 4} \leq \frac{1}{1+\frac{1}{2^{S+1}}} \omega_{\rho}
$$

Now we consider the case $\operatorname{dist}\left(x_{0}, \Gamma_{D}^{u}\right)<\rho$. Since $\operatorname{osc}_{\Gamma_{D}^{u} \cap \bar{Q}_{2 \rho}} u_{0} \leq C \rho$, we have that either $M_{\rho} \geq M_{2 \rho}^{0}+\frac{1}{4} \omega_{\rho}$ or $m_{\rho} \leq m_{2 \rho}^{0}-\frac{1}{4} \omega_{\rho}$. In both cases we can apply Lemma 2.6, Lemma 2.8 and Lemma 2.9 to conclude that

$$
\omega_{\rho / 2} \leq \frac{1}{1+\frac{1}{2^{s+1}}} \omega_{2 \rho}
$$


This concludes the assertion of the Lemma.

Finally the assertion of Theorem 2.1 follows from Lemma 2.10 and Lemma 5.8, p. 96 of [10].

\section{A priori estimates}

We establish here some a priori estimate for the solution of the system (1.1)-(1.7), under the assumptions

$D^{\prime}$ is Lipschitz continuous, $\quad D \geq D_{m}>0 ;$

$K^{\prime}$ is Lipschitz continuous, $K \geq K_{m}>0$;

$$
\begin{gathered}
p_{1} \in C^{2}\left(\bar{Q}_{T}\right), \quad \varphi_{0} \in H^{2+\alpha}(\bar{\Omega}) \\
u_{0} \in H^{2+x}(\bar{\Omega}), \quad \frac{\partial u_{0}}{\partial n}=0, \quad x \in \partial \Omega .
\end{gathered}
$$

As a first step, we prove

Lemma 3.1. Under assumptions (3.1)-(3.4), we have

$$
\begin{gathered}
|u| \leq M, \quad(x, t) \in Q_{T} \\
\|\nabla u\|_{L^{2}\left(Q_{T}\right)} \leq M, \\
\|p(\cdot, t)\|_{L^{2}(\Omega)}+\|\nabla p(\cdot, t)\|_{L^{2}(\Omega)} \leq M, \quad \text { for every } t \in[0, T], \\
|\varphi|+\left|\varphi_{t}\right| \leq M, \quad(x, t) \in Q_{T}, \\
\|\nabla \varphi(\cdot, t)\|_{L^{2}(\Omega)} \leq M, \quad \text { for every } t \in[0, T],
\end{gathered}
$$

Proof. Inequality (3.5) is a standard consequence of the maximum principle and inequality (3.8) follows immediately from (3.5) and equation (1.3). Inequalities (3.6) and (3.7) are easily obtained multiplying equation (1.1) times $u$ and equation (1.2) times $p$ and then integrating by part, see Lemma 4.1 of [7] for more details.

Lemma 3.2. Under assumptions (3.1)-(3.4), we have the following a priori estimates

$$
\begin{gathered}
|u|_{\hat{Q}_{T}}^{(2+\alpha)} \leq M, \\
|p|_{\bar{Q}_{\tau}}^{(\delta)}+|\nabla p|_{\bar{Q}_{T}}^{(\delta)} \leq M(\delta), \quad \text { for any } \delta \in(0,1), \\
\left|\varphi_{t}\right|_{\hat{Q}_{T}}^{(2+\alpha)}+|\nabla \varphi|_{\bar{Q}_{T}}^{(1+\alpha)}+\left|\nabla \varphi_{t}\right|_{\bar{Q}_{T}}^{(1+\alpha)} \leq M .
\end{gathered}
$$


Moreover $p \in L^{\infty}\left(0, T ; H^{2+\alpha}(\omega)\right)$ for any $\omega \subset \subset \Omega$.

Proof. Writing equation (1.1) as

$$
\varphi \frac{\partial}{\partial t} u=\nabla(D \nabla u)+K \nabla p \nabla u+\frac{\partial}{\partial t} \varphi
$$

we can apply, because of the estimates of Lemma 3.1, the results of Section 2, thus obtaining the Hölder continuity of $u$ in $\bar{Q}_{T}$. Consequently also $\varphi$ and $\varphi_{t}$ are Hölder continuous functions.

Then using the regularity result of Theorem 17.1 of [2], we have

$$
|p(\cdot, t)|_{\Omega}^{(1+\beta)} \leq M
$$

for some $\beta \in(0,1)$, for every $t \in(0, T)$.

Then we proceed as in the proof of Theorem 2.3 of [4], i.e. for every positive constants $h$ and $\gamma$, we denote by $p_{\gamma, h}$ the difference $(p(\cdot, t+h)-p(\cdot, t)) / h^{\gamma}$. Then $p_{\gamma, h}$ satisfies the elliptic equation

$$
\nabla\left(K(\varphi(\cdot, t+h)) \nabla p_{\gamma, h}\right)=\left(\varphi_{t}\right)_{y, h}+\nabla\left(K(\varphi)_{y, h} \nabla p\right)
$$

Since $K(\varphi)_{\gamma, h}$ is Hölder continuous, uniformly in $h$, for every $\gamma \in[0,1]$, we then know that $\nabla p_{\alpha / 2, h}$ is bounded independently of $h,[2]$. Hence, we obtain

$$
|p|_{\bar{Q}_{T}}^{(\beta)}+|\nabla p|_{\bar{Q}_{T}}^{(\beta)} \leq M .
$$

Again, we proceed as in part (d) of the proof of the above mentioned theorem of [4], i.e. differentiating equation (3.13) and multiplying by $|\nabla u|^{2 s} u_{x_{i}}$, summing over $i$ and integrating by parts (noting that $\partial u / \partial n=0$ on $\partial \Omega_{M} \times(0, T)$ ), we get

$$
\begin{aligned}
0= & \int_{0}^{T} \iint_{\Omega_{M}}\left\{\left[\frac{1}{2(1+s)} \varphi|\nabla u|^{2 s+2}\right]_{t}+D u_{x_{i} x_{j}}\left(|\nabla u|^{2 s} u_{x_{i}}\right)_{x_{j}}\right. \\
& -\frac{1}{2(1+s)} \varphi_{t}|\nabla u|^{2 s+2}+\varphi_{x_{i}} u_{t}|\nabla u|^{2 s} u_{x_{i}}+D_{x_{i}} u_{x_{j}}\left(|\nabla u|^{2 s} u_{x_{i}}\right)_{x_{j}} \\
& \left.+K \nabla u \nabla p\left(|\nabla u|^{2 s} u_{x_{i}}\right)_{x_{i}}-\left(\varphi_{t}\right)\left(|\nabla u|^{2 s} u_{x_{i}}\right)_{x_{i}}\right\}
\end{aligned}
$$

Here we have used the conventional summation notation over doubled indices. The first two terms in the integrand are good terms which will give the positive terms $\|\nabla u(\cdot, t)\|_{L^{2+2}\left(\Omega_{M}\right)}^{2 s+2}$ and $\left\||\nabla u|^{s}\left|\left(u_{x_{i} x_{j}}\right)\right|\right\|_{L^{2}\left(\Omega_{M, t}\right)}^{2}$, where we indicate by $\left|\left(u_{x_{i} x_{j}}\right)\right|$ the euclidean norm of the Hessian matrix in $\mathbf{R}^{N \times N}$. Using the equation to replace $u_{t}$, the fourth term in the integrand can be estimated by $\int_{0}^{t} \iint_{\Omega_{M}}\left(\epsilon|\nabla u|^{2 s}\left|\left(u_{x_{i} x_{j}}\right)\right|^{2}+C_{\varepsilon}\left(1+|\nabla u|^{2 s+4}+|\nabla \varphi|^{2 s+4}\right)\right)$. 
Similar estimates also hold for the rest of the terms. Since $\|\nabla \varphi\|_{L^{2+4}\left(\Omega_{M, l}\right)} \leq$ $C\left(1+\|\nabla u\|_{L^{2+4}\left(\Omega_{M, t}\right)}\right)$, taking $\epsilon$ small enough, we obtain

$$
\sup _{0 \leq r \leq t}\left\||\nabla u|^{s+1}\right\|_{L^{2}\left(\Omega_{M}\right)}^{2}+\left\||\nabla u|^{s}\left|\left(u_{x_{i} x_{j}}\right)\right|\right\|_{L^{2}\left(\Omega_{M, t}\right.}^{2} \leq C(s)\left[1+\int_{0}^{t} \iint_{\Omega_{M}}|\nabla u|^{2 s+4}\right] .
$$

Since $u \in C^{x, \alpha / 2}$ and $\partial u / \partial n=0$ on the boundary, using a reflection technique we can rewrite the imbedding of $[10,(5.8)$, p. 94$]$ in the form:

$$
\iint_{\Omega_{M}}|\nabla u|^{2 s+4} \leq \iint_{\Omega_{M}} \epsilon|\nabla u|^{2 s}\left|\left(u_{x_{i} x_{j}}\right)\right|^{2}+C_{\epsilon}|\nabla u|^{2 s+2}
$$

from which it follows that

$$
\sup _{0 \leq r \leq t}\left\||\nabla u|^{s+1}\right\|_{L^{2}\left(\Omega_{M}\right)}^{2}+\left\||\nabla u|^{s}\left|\left(u_{x_{i} x_{j}}\right)\right|\right\|_{L^{2}\left(\Omega_{M, t}\right)}^{2} \leq C(s)\left[1+\int_{0}^{t} \iint_{\Omega_{M}}|\nabla u|^{2 s+2}\right] .
$$

Gronwall's inequality then gives that $\nabla u \in L^{\infty}\left(0, T ; L^{2 s+2}\left(\Omega_{M}\right)\right)$ for any $s \geq 0$.

We thus obtain an estimate for the $L^{s}\left(Q_{T}\right)$-norm of $|\nabla u|$ for any $s$. Such an estimate induces a similar estimate for $\nabla \varphi$. Then we can consider 3.13 as a linear equation whose coefficients are bounded in appropriate spaces. Theorem 9.1, p. 341, of [10] and the subsequent corollary can now be applied, and we obtain that

$$
\|u\|_{q, Q_{T}}^{(2)}+|u|_{\tilde{Q}_{T}}^{(1+\gamma)} \leq M(q, \gamma)
$$

for any $q \geq 2$ and any $\gamma \in(0,1)$.

The statement of the lemma then follows from standard regularity theorems on linear elliptic and parabolic equations by means of a simple boot-strap argument.

We assume now that $u_{0}$ satisfies the following weaker condition.

$$
u_{0} \in W_{r}^{2-(N+2) / r}(\bar{\Omega}), \quad r>N+2, \quad \frac{\partial u_{0}}{\partial n}=0, \quad x \in \partial \Omega
$$

Theorem 3.3. Under the assumptions (3.1), (3.3) and (3.15), problem (1.1)-(1.7) has a unique classical solution $(u, p, \varphi)$ such that

$$
\begin{array}{r}
u \in W_{r}^{2}\left(\bar{Q}_{T}\right) \cap H^{2-(N+2) / r}\left(\bar{Q}_{T}\right), \\
\varphi \in C^{2-(N+2) / r}\left(\bar{Q}_{T}\right), \\
p \in L^{\infty}\left(0, T ; H^{2+v}(\omega)\right) \quad \omega \subset \subset \Omega,
\end{array}
$$

for a suitable $v \in(0,1)$. Moreover $p$ satisfies (3.11), $u$ and $\varphi$ satisfy inequalities (3.10) and (3.12) in $\overline{\Omega \times(\tau, T)}$ for any $\tau>0$. 
Proof. Local existence and uniqueness of a classical solution satisfying (3.16) and (3.17) is granted by Remark 1.2 of [7] (in [7] a Dirichlet datum was assigned for $u$, but the arguments used there work also for a Neumann boundary condition).

Standard regularity theory implies that this solution satisfies the assumptions on the initial data of Lemma 3.2 for any positive $t$ in the interval of existence. The a priori estimates of Lemma 3.2 then guarantee that the solution can be extended for arbitrary positive time preserving the regularity required in (3.16)-(3.18) ((3.18) is a trivial consequence of elliptic regularity theory, [9]).

Remark 3.1. The previous results remain true under the boundary condition $(\partial u / \partial n)=h(x, t)$, with $h$ regular enough, providing that an estimate for $|u|$ is available.

\section{REFERENCES}

1. H. W. Alt and E. DiBenedetto. Nonsteady flow of water and oil through inhomogeneous porous media, Ann. Scuola Norm. Sup. Pisa, IV 12 (1985), 335-392.

2. S. Campanato, Equazioni ellittiche del II ordine e spazi $\mathcal{L}^{(2, \lambda)}$, Ann. Mat. Pura Appl. 69 (1965), 321-380.

3. J. Chadam, Xinfu Chen, E. Comparini and R. Ricci, Traveling wave solutions of a reaction-infiltration problem and a related free boundary problem, European J. Appl. Math., to appear.

4. J. Chadam, Xinfu Chen, R. Gianni and R. Ricci, A reaction infiltration problem: Existence, uniqueness, and regularity of solutions in two space dimensions, Math. Models Methods Appl. Sci., to appear.

5. J. Chadam, D. Hoff, E. Merino, P. Ortoleva and A. Sen, Reactive Infiltration Instabilities, IMA J. Appl. Math. 36 (1987), 207-220.

6. E. DiBenedetto, in Theory and Applications of Liquid Cristal (IMA, Vol. 5), 123-141.

7. R. GianNi and R. Ricci, Existence and Uniqueness for a Reaction-Diffusion Problem in Infiltration, Ann. Mat. Pura Appl., to appear.

8. M. GIAQUINTA, Multiple Integrals in the Calculus of Variations and Nonlinear Elliptic Systems (Princeton University Press, Princeton, New Jersey, 1983).

9. G. Gilbarg and N. S. Trudinger, Elliptic Partial Differential Equations of Second Order (Springer, Berlin, 2nd ed., 1983).

10. O. A. Ladyženskaja, V. A. Solonnikov and N. N. Ural'Ceva, Linear and Quasilinear Equations of Parabolic Type (Translations of Mathematical Monographs, 23, Providence R.I., American Mathematical Society, 1968).

11. O. A. LADYžEnSKajA and N. N. URAL'CEVA, Equations aux dérivées partielles de type elliptique (Dunod, Paris, 1968). 
A REACTION INFILTRATION PROBLEM: CLASSICAL SOLUTIONS 291

FieldS INSTITUTE FOR RESEARCH

in the Mathematical SCienCes

185 Columbia Street

WATERLOO

ONTARIO N2L $5 Z 5$

Canada

Permanent address:

Department of Mathematics and Statistics

MCMASTER UNIVERSITY

HAMILTON

ONTARIO L8S 4K1

CANADA

Dipartimento di Matematica

UNIVERSITA DI FIRENZE

FLORENCE

ITALY
Department of Mathematics

University of PitTsburgh

Pittsaurgh

Pennsylvania 15260

U.S.A.

Dipartimento di Matematica UNIVERSITA DI MILANO

Milan

ITALY 